\title{
One-pot synthesis of m-terphenyl-2'-carbaldehydes
}

\author{
Bahaaldin Rashidzadeh, ${ }^{a}$ Farnaz Jafarpour, ${ }^{\mathrm{b}}$ and Akbar Saednya ${ }^{\mathrm{c}}$ \\ ${ }^{a}$ Payame Noor University, Department of Chemistry, PO Box 66815-335, Saghez, Iran \\ ${ }^{\mathrm{b}}$ School of Chemistry, University College of Science, University of Tehran, PO Box 14155-6455, \\ Tehran, Iran \\ ${ }^{\mathrm{c}}$ Department of Organic Chemistry, Faculty of Chemistry, Tabriz University, Tabriz 51664, Iran \\ E-mail: jafarpur@khayam.ut.ac.ir
}

\begin{abstract}
An efficient and straightforward one pot synthesis of $m$-terphenyl-2'-carbaldehyde derivatives via cascade reaction of aryl Grignard magnesium bromide with 1,3-dichloroiodobenzene is described. Via the reactions three carbon-carbon bonds are constructed and m-terphenyls are obtained in good yields.
\end{abstract}

Keywords: One pot synthesis, cascade reaction, $m$-terphenyl-2'-carbaldehyde, 1,3dichloroiodobenzene, carbon-carbon bond construction

\section{Introduction}

The m-terphenyl moiety is an important structure for concave molecules. ${ }^{1}$ If a functional group is introduced into the 2'-position of a m-terphenyl, the outer benzene rings shield this functional group. The shielding is extremely efficient if the outer benzene rings carry further substituents in the ortho positions (Figure 1).

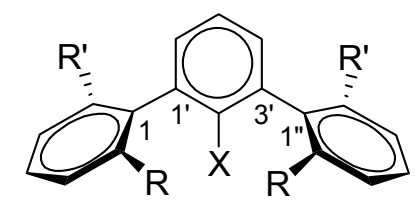

\section{Figure 1}

Some m-terphenyls with acidic substituents in the 2'-position have been introduced as selective proton donors for diastereoselective and regioselective protonations. ${ }^{2}$ In addition, these compounds are useful intermediates for constructing optical active cyclophans, ${ }^{3,4}$ cyclic ketons, ${ }^{5-8}$ 
dendrimers, ${ }^{9}$ and liquid crystals. ${ }^{10}$ Thus, several approaches have been developed for their synthesis. Hart developed a method for the synthesis of m-terphenyls that may be substituted in all orthopositions of the outer aryl rings. ${ }^{11,12}$ The key intermediate of Hart's method is the 2'-Grignard reagent, which can be quenched by iodine to give the 2 '-iodide substituted compound. Starting from the iodide compound, various 2 -substituted $m$-terphenyls have been synthesized via the lithium derivative. Limited studies were carried out with aryl magnesium halides and other 1,2,3trihalobenzenes such as 2,6-dichloroiodobenze and only moderate yields of the products were obtained. $^{4,11-13}$ Furthermore, most reports on the synthesis of 2'-substituted m-terphenyl derivatives are limited to the two step procedure (Scheme 1).<smiles>Brc1cccc(Br)c1I</smiles><smiles>FC(Br)C(F)(Br)Br</smiles>

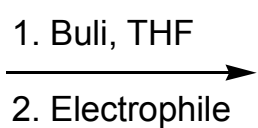

3. $\mathrm{H}_{3} \mathrm{O}^{+}$

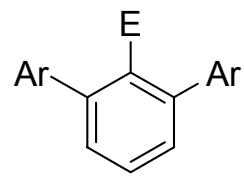

Scheme 1. Synthesis of 2'-substituted m-terphenyl derivatives via two step procedure.

In the current study, we examine a facile one pot synthesis of m-terphenyl-2'-carbaldehydes starting from 1,3-dichloroiodobenzene. Cascade reaction of aryl magnesium bromides with 1,2,3-trihalobenzene $\mathbf{1}$ followed by quenching with electrophile gives m-terphenyls $\mathbf{2}$ in a one pot reaction. By varying the substituents of the aryl groups of the Grignard reagent, different $\mathrm{m}$ terphenyl derivatives can be obtained in good yields.

\section{Results and Discussion}

Our initial attempts to test the feasibility of this reaction employed readily accessible 1,3dichloroiodobenzene and phenyl magnesium bromide in THF which afforded 2,3-diphenyl magnesium bromide reagent. Then the addition of ethyl formate gave the desired product $\mathbf{2 a}$ in $81 \%$ yield (Table 1). We next investigated the scope of the reaction using aryl magnesium bromides with diverse substituents at ortho-, meta- and para-positions. As a result, m-terphenyl2'-carbaldehyde derivatives were obtained in good yields (Table 1). The structures of the isolated products were confirmed by mp, IR, ${ }^{1} \mathrm{HNMR},{ }^{13} \mathrm{CNMR}$ and elemental analysis.

It should be mentioned that ortho-substituted m-terphenyls which can be further functionalized or converted to new functional groups are very important. For example, methoxy groups of the rings can be converted easily to phenol derivatives and bridged to finally give bimacrocylic concave m-terphenyl structures. Further investigation on involving these $\mathrm{m}$ terphenyls in the polymer backbones is under investigation. 
Table 1. One pot synthesis of m-terphenyl-2'-carbaldehyde derivatives

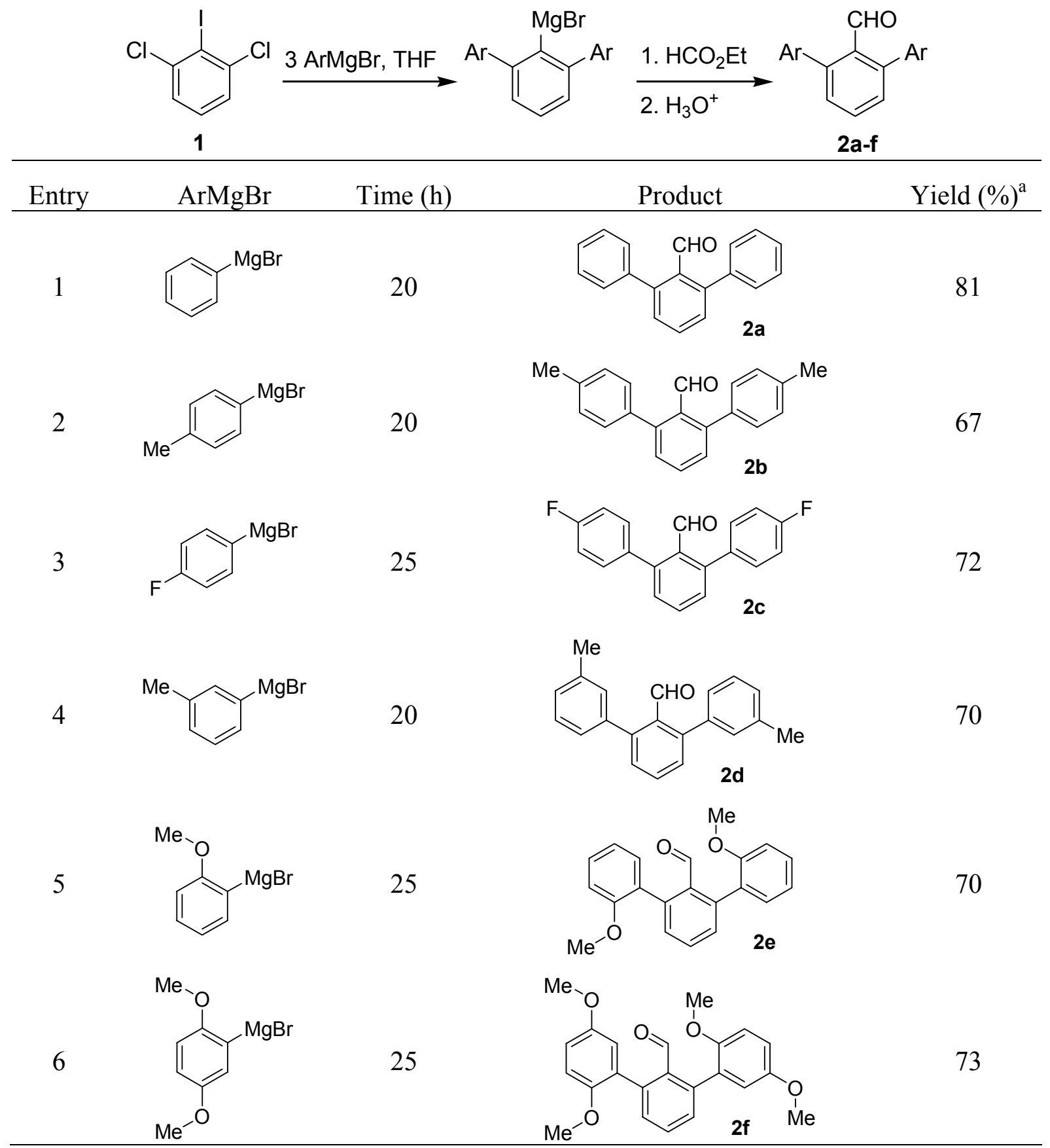

${ }^{\text {a }}$ Isolated yields 


\section{Conclusions}

In summary, we have developed an efficient and straightforward one pot approach to mterphenyl-2'-carbaldehyde derivatives using readily accessible starting materials. To the best of our knowledge it is the first report on the one pot synthesis of m-terphenyl-2'-carbaldehyde derivatives.

\section{Experimental Section}

General Procedures. All substrates were purchased from Merck Company. The reactions involving organomagnesium halide reagents were carried out under argon. Solvents were dried by standard methods. Melting points were determined using a Gallenkamp melting point apparatus and are uncorrected. ${ }^{1} \mathrm{H}$ NMR and ${ }^{13} \mathrm{C}$ NMR spectra were recorded on a Brucker FT$400 \mathrm{MHz}$ in $\mathrm{CDCl}_{3}$. The Infrared absorption spectra were recorded on a FTIR Bruker-Tensor 270 spectrophotometer. Elemental analyses were carried out with Heareus CHN-RAPID instrument.

General procedure for the synthesis of m-terphenyl-2'-carbaldehyde derivatives 2a-2f

To a stirred aryl magnesium bromide solution (prepared from aryl bromides $(40 \mathrm{mmol})$ and $\mathrm{Mg}$ $(40 \mathrm{mmol})$ in dry THF $(40 \mathrm{~mL}))$ was added slowly 1,3-dichloroiodobenzene $(4 \mathrm{mmol})$ in THF $(10 \mathrm{~mL})$ under argon and the mixture was refluxed for $20-25 \mathrm{hrs}$. Then ethyl formate $(5 \mathrm{~mL})$ was added and the reaction was refluxed for $30 \mathrm{~min}$. The reaction mixture was then cooled with ice, quenched with dilute $\mathrm{HCl}(30 \mathrm{~mL})$ and extracted with diethyl ether $(3 \times 50 \mathrm{~mL})$. Combined organic layers were dried over $\mathrm{MgSO}_{4}$ and concentrated in vacuo. The resulting residue was purified by recrystallization or column chromatography (diethyl ether- hexane, $20 \%$ ) to afford the corresponding aldehydes.

1, $\mathbf{1}^{\prime}, \mathbf{3}^{\prime}, \mathbf{1}^{\prime \prime}$-Terphenyl-2'-carbaldehyde (2a). Mp 76-77 $\mathrm{C}$; IR (KBr, $\left.\mathrm{cm}^{-1}\right)$ : 2850, 2750, 1700, 1600,$1450 ;{ }^{1} \mathrm{H}$ NMR $\left(400 \mathrm{MHz}, \mathrm{CDCl}_{3}\right): \delta 9.65(\mathrm{~s}, 1 \mathrm{H}), 7.28(\mathrm{t}, J=7.6 \mathrm{~Hz}, 1 \mathrm{H}), 7.09(\mathrm{~m}, 12 \mathrm{H})$; ${ }^{13} \mathrm{C}$ NMR $\left(100 \mathrm{MHz}, \mathrm{CDCl}_{3}\right): \delta 194.0,144.7,140.1,133.6,131.9,130.8,130.0,128.6,128.1$; Anal. Calcd for $\mathrm{C}_{19} \mathrm{H}_{14} \mathrm{O}$ : C, 88.34; H, 5.46. Found: C, 88.42; H, 5.51.

4,4"-Dimethyl-1,1',3', $\mathbf{1}^{\prime \prime}$-terphenyl-2'-carbaldehyde (2b). Mp 108-109 ${ }^{\circ} \mathrm{C}$. IR $\left(\mathrm{KBr}, \mathrm{cm}^{-1}\right)$ : 2850, 2750, 1700, 1600, 1450; ${ }^{1} \mathrm{H}$ NMR (400 MHz, $\left.\mathrm{CDCl}_{3}\right): \delta 10.00$ (s, $\left.1 \mathrm{H}\right), 7.59$ (t, $J=7.6 \mathrm{~Hz}$, $1 \mathrm{H}), 7.40(\mathrm{~d}, J=7.6 \mathrm{~Hz}, 2 \mathrm{H}), 7.28(\mathrm{~m}, 8 \mathrm{H}), 2.45(\mathrm{~s}, 6 \mathrm{H}) ;{ }^{13} \mathrm{C} \mathrm{NMR}\left(100 \mathrm{MHz}, \mathrm{CDCl}_{3}\right): \delta 194.4$, 144.7, 137.9, 137.1, 133.6, 131.9, 130.6, 129.9, 129.3, 21.7; Anal. Calcd for $\mathrm{C}_{21} \mathrm{H}_{18} \mathrm{O}: \mathrm{C}, 88.08$; H, 6.34. Found: C, 87.90; H, 6.40.

4,4"-Fluoro-1,1',3',1"'-terphenyl-2'-carbaldehyde (2c). Mp 152-153 ${ }^{\circ} \mathrm{C}$. IR $\left(\mathrm{KBr}, \mathrm{cm}^{-1}\right)$ : 2850 , 2750, 1700, 1600, 1450; ${ }^{1} \mathrm{H}$ NMR $\left(400 \mathrm{MHz}, \mathrm{CDCl}_{3}\right): \delta 9.61(\mathrm{~s}, 1 \mathrm{H}), 7.28(\mathrm{t}, J=7.6 \mathrm{~Hz}, 1 \mathrm{H}$ ), $7.06(\mathrm{~d}, J=7.6 \mathrm{~Hz}, 2 \mathrm{H}), 7.01(\mathrm{~m}, 4 \mathrm{H}), 6.82(\mathrm{~m}, 4 \mathrm{H}) ;{ }^{13} \mathrm{C} \mathrm{NMR}\left(100 \mathrm{MHz}, \mathrm{CDCl}_{3}\right): \delta 193.6$, 
164.2, 161.7, 143.8, 135.9, 135.8, 133.5, 132.1, 131.7, 131.6, 131.0, 115.7, 115.5; Anal. Calcd for $\mathrm{C}_{19} \mathrm{H}_{12} \mathrm{~F}_{2} \mathrm{O}: \mathrm{C}, 77.54 ; \mathrm{H}, 4$.11. Found: C, 77.48; H, 4.20.

3,3"-Dimethyl-1,1',3',1"-terphenyl-2'-carbaldehyde (2d). Mp 95-96 ${ }^{\circ} \mathrm{C}$. IR ( $\left.\mathrm{KBr}, \mathrm{cm}^{-1}\right): 2850$, 2750, 1700, 1600, 1450; ${ }^{1} \mathrm{H}$ NMR $\left(400 \mathrm{MHz}, \mathrm{CDCl}_{3}\right): \delta 10.00(\mathrm{~s}, 1 \mathrm{H}), 7.62(\mathrm{t}, J=7.6 \mathrm{~Hz}, 1 \mathrm{H})$, $7.41(\mathrm{~d}, J=7.7 \mathrm{~Hz}, 2 \mathrm{H}), 7.35(\mathrm{t}, J=7.5 \mathrm{~Hz}, 2 \mathrm{H}), 7.25$ (d, $J=7.6 \mathrm{~Hz}, 2 \mathrm{H}), 7.21(\mathrm{~s}, 2 \mathrm{H}), 7.18$ (d, $J=7.5 \mathrm{~Hz}, 2 \mathrm{H}), 2.45(\mathrm{~s}, 6 \mathrm{H}) ;{ }^{13} \mathrm{C} \mathrm{NMR}\left(100 \mathrm{MHz}, \mathrm{CDCl}_{3}\right): \delta 194.3,144.8,140.1,138.3,133.6$, 131.9, 130.7, 128.8, 128.5, 127.2, 21.9; Anal. Calcd for $\mathrm{C}_{21} \mathrm{H}_{18} \mathrm{O}$ : C, 88.08; H, 6.34. Found: C, 87.80; H, 6.30.

2,2"'-Dimethoxy-1,1',3',1"-terphenyl-2'-carbaldehyde (2e). Mp $165-166^{\circ} \mathrm{C}$. IR ( $\left.\mathrm{KBr}, \mathrm{cm}^{-1}\right)$ : 2850, 2750, 1700, 1600, 1450; ${ }^{1} \mathrm{H}$ NMR (400 MHz, $\mathrm{CDCl}_{3}$ ): $\delta 9.79$ (s, 1H), 7.66 (t, J= $7.6 \mathrm{~Hz}$, $1 \mathrm{H}), 7.42(\mathrm{t}, J=7.4 \mathrm{~Hz}, 4 \mathrm{H}), 7.36(\mathrm{~d}, J=7.6 \mathrm{~Hz}, 2 \mathrm{H}), 7.13(\mathrm{~d}, J=7.6 \mathrm{~Hz}, 2 \mathrm{H}), 6.96(\mathrm{~d}, J=7.6$ $\mathrm{Hz}, 2 \mathrm{H}), 3.79$ (s, 6H); ${ }^{13} \mathrm{C} \mathrm{NMR}\left(100 \mathrm{MHz}, \mathrm{CDCl}_{3}\right): \delta 193.0,156.4,139.4,132.6,131.2,131.0$, 129.8, 129.5, 129.3, 121.3, 110.9, 55.7; Anal. Calcd for $\mathrm{C}_{21} \mathrm{H}_{18} \mathrm{O}_{3}$ : C, 79.22; H, 5.70. Found: C, $79.41 ; \mathrm{H}, 5.81$.

2,2",5,5"-Tetramethoxy-1,1',3',1"-terphenyl-2'-carbaldehyde (2f). Mp 186- $187^{\circ} \mathrm{C}$. IR ( $\mathrm{KBr}$, $\left.\mathrm{cm}^{-1}\right): 2850,2750,1700,1600,1450 ;{ }^{1} \mathrm{H}$ NMR (400 MHz, $\left.\mathrm{CDCl}_{3}\right): \delta 9.75(\mathrm{~s}, 1 \mathrm{H}), 7.64(\mathrm{t}, J=$ $7.6 \mathrm{~Hz}, 1 \mathrm{H}), 7.35$ (d, $J=7.6 \mathrm{~Hz}, 2 \mathrm{H}), 7.03(\mathrm{~s}, 2 \mathrm{H}), 6.92$ (d, $J=7.6 \mathrm{~Hz}, 2 \mathrm{H}), 6.86$ (d, $J=7.6 \mathrm{~Hz}$, 2H), $3.85(\mathrm{~s}, 6 \mathrm{H}), 3.68(\mathrm{~s}, 6 \mathrm{H}) ;{ }^{13} \mathrm{C} \mathrm{NMR}\left(100 \mathrm{MHz}, \mathrm{CDCl}_{3}\right): \delta 192.7,154.0,153.4,152.1$, $150.5,139.0,132.5,130.9,130.0,117.2,114.0,111.8,111.3,56.1$; Anal. Calcd for $\mathrm{C}_{23} \mathrm{H}_{22} \mathrm{O}_{5}$ : C, 73.00; H, 5.86. Found: C, 72.64; H, 5.92.

\section{Acknowledgements}

We thank the Research Council of the University of Tabriz and University of Tehran for financial support for this project $(27827 / 1 / 01)$.

\section{References}

1. Abbass, M.; Kuhl, C.; Manthey, C.; Muller, A.; Luning, U. Collect. Czech. Chem. Commun. 2004, 69, 1325.

2. Luning, U.; Baumgartner, H.; Manthey, C.; Meynhardt, B. J. Org. Chem. 1996, 61, 7922.

3. Vinod, T. K.; Rajakumar, P.; Hart, H. Tetrahedron 1995, 51, 2267.

4. Hart, H.; Rajakumar, P. Tetrahedron 1995, 51, 1313.

5. Viond, T. K.; Hart, H. J. Org. Chem. 1990, 55, 881.

6. Rajakumar, P.; Kannan, A. Tetrahedron lett. 1993, 34, 4407.

7. Grewal, R.S.; Hart, H.; Viond, T. K. J. Org. Chem. 1992, 57, 2721.

8. Kei, G.; Gaku, Y. Tetrahedron Lett. 2001, 42, 4875.

9. Rajakumar, P.; Srisailas, M. Tetrahedron Lett. 1997, 38, 5323. 
10. Rajakumar, P.; Srisailas, M. Tetrahedron 2001, 57, 9749.

11. Du, C.-J. F.; Hart, H.; Ng, K.-K. D. J. Org. Chem. 1986, 51, 3162.

12. Vinod, T. K.; Hart, H. Top. Curr. Chem. 1994, 172, 119.

13. Du, C.-J. F.; Hart, H. J. Org. Chem. 1987, 52, 4311. 